\title{
Concomitant Chronic Inflammatory Demyelinating Polyradiculoneuropathy and Myasthenia Gravis-A Two-Case Report
}

\section{Nan Jiang* and Mohamed Kazamel}

Division of Neuromuscular Disease, Department of Neurology, The University of

Alabama at Birmingham, Birmingham, Alabama, United States of America

*Corresponding Author: Nan Jiang, Division of Neuromuscular Disease, Department of Neurology, The University of Alabama at Birmingham, Birmingham, Alabama, United States of America.
Received: August 12, 2020

Published: August 31, 2020

(C) All rights are reserved by Nan Jiang and

Mohamed Kazamel.

\begin{abstract}
Few cases of co-existing chronic inflammatory demyelinating polyradiculoneuropathy (CIDP) and seropositive myasthenia gravis (MG) were reported. However, there is no reports on co-existence of CIDP with seronegative MG or on the responses to treatment of both diseases. We report two cases with diagnosis of CIDP and MG. The first case is 38-year-old African American female with initial diagnosis of CIDP and later onset bulbar symptoms. She was diagnosed with seronegative MG by clinical suspicion and single fiber electromyography (SFEMG). The other case is a 65-year-old British male who initially diagnosed as seropositive MG then he developed paresthesia and difficult walking a few months later. CIDP was confirmed by electrodiagnostic study and albuminocytologic dissociation in cerebrospinal fluid analysis. The first case underscores the importance of performing SFEMG on cranial muscles in cases of CIDP with cranial nerve involvement even if serological testing for MG is negative. In both cases, bulbar symptoms were more responsive to immunomodulatory treatment than limb symptoms. .
\end{abstract}

Keywords: Chronic Inflammatory Demyelinating Polyradiculoneuropathy; Immunomodulatory Treatment; Myasthenia Gravis; Single Fiber EMG; Treatment Response

\section{Abbreviations}

AchR: Acetylcholine Receptor; CIDP: Chronic Inflammatory Demyelinating Polyradiculoneuropathy; CSF: Cerebrospinal Fluid; CT: Computed Tomography; EMG: Electromyography; IVIg: Immunoglobulin Infusion; MG: Myasthenia Gravis; MRC: Medical Research Council; MusK: Muscle-Specific Kinase; NCS: Nerve Conduction Study; RNS: Repetitive Nerve Stimulation Study; SFEMG: Single Fiber Electromyography; WBC: White Blood Cell Count

\section{Introduction}

Chronic inflammatory demyelinating polyradiculoneuropathy (CIDP) and myasthenia gravis (MG) are very distinct autoimmune disorders when it comes to pathology and pathophysiology $[1,2]$.
The concomitance of both diseases was reported few times in different scenarios [3-7]. Here, we report two cases with concomitant CIDP and MG (seropositive and seronegative), and the different responses to treatment of each disease in the two cases.

\section{Case Report}

Case 1

A 38-year-old African American female patient without significant medical history who presented with progressive sensory deficit and followed by lower limb weakness two months later. She developed left ptosis, diplopia, mild dysphagia, chewing fatigue, and dyspnea six months after the onset of limb symptoms. Neurologic examination revealed facial weakness, and distal greater than 
proximal symmetric weakness in upper and lower limbs. Medical Research Council (MRC) grades were: elbow flexion 4/5, wrist extension 4-/5, finger extension 3/5, dorsal interossei 3/5, knee flexion 5-/5, ankle dorsiflexion $3 / 5$ and plantar flexion $4 / 5$ bilaterally. Sensory examination reveals reduced pinprick sensation at both hands and feet, reduced vibration sense at knees and absent at big toes with impaired proprioceptive testing at both big toes and ankles. Deep tendon reflexes were absent throughout. She was unable to walk without assistance. Nerve conduction studies (NCS) demonstrated significant acquired demyelinating features in the tested motor nerves (Table 1), in addition to evidence of polyradiculopathy and secondary axonal damage in EMG, which is consistent with CIDP. Repetitive nerve stimulation study (RNS) was unremarkable. Acetylcholine receptor (AchR) and MusK (Muscle-specific kinase) antibodies were negative. Single fiber electromyography (SFEMG) of the left frontalis muscle showed five outlying pairs $(83 \%)$ with jitter values greater than the $95 \%$ upper confidence limit expected for age with mean consecutive difference of $72.3 \mu \mathrm{s}$. Immunoglobulin infusion (IVIg) was discontinued after one dose because of aseptic meningitis. Bulbar symptoms improved significantly with treatment of prednisone $60 \mathrm{mg}$ daily for 2 weeks and $40 \mathrm{mg}$ daily for 3 weeks and pyridostigmine, However, limbs muscle strength did not improved even with plasmapheresis. She is currently off prednisone and other immunosuppressant with minimal bulbar and ocular symptoms but significant stable both proximal and distal limb weakness.

\begin{tabular}{|l|c|c|c|}
\hline $\begin{array}{l}\text { Nerve condition } \\
\text { study }\end{array}$ & Case 1 & Case 2 & $\begin{array}{c}\text { UAB } \\
\text { NL }\end{array}$ \\
\hline Motor & & & \\
\hline Peroneal & 11.3 & NR & $<4.8$ \\
\hline Distal latency (ms) & $1.4,0.7,0.4^{* * *}$ & & $>2.5$ \\
\hline CAMP (mV) & $18,33^{* *}$ & & $>40$ \\
\hline $\begin{array}{l}\text { Conduction velocity } \\
\text { (m/s) }\end{array}$ & NR & & $<54$ \\
\hline F wave latency (ms) & 13.1 & & $<6.0$ \\
\hline Tibial & $0.2,0.1^{* * *}$ & & $>4.0$ \\
\hline Distal latency (ms) & 25 & & \\
\hline CAMP (mV) & & & \\
\hline $\begin{array}{l}\text { Conduction velocity } \\
\text { (m/s) }\end{array}$ & & & \\
\hline
\end{tabular}

\begin{tabular}{|c|c|c|c|}
\hline $\mathrm{F}$ wave latency (ms) & NR & & $<55$ \\
\hline \multicolumn{4}{|l|}{ Median } \\
\hline Distal latency (ms) & 6.3 & $4.7(<4.3)^{*}$ & $<3.5$ \\
\hline CAMP $(\mathrm{mV})$ & $2.4,1.6^{* * *}$ & $2.7,1.7(>4)^{*}$ & $>5.0$ \\
\hline $\begin{array}{l}\text { Conduction velocity } \\
(\mathrm{m} / \mathrm{s})\end{array}$ & 22 & $31.4(>49)^{*}$ & $>50$ \\
\hline F wave latency (ms) & 76.84 & $51.3(<32)^{*}$ & $<31$ \\
\hline \multicolumn{4}{|l|}{ Right Ulnar } \\
\hline Distal latency (ms) & 5.2 & $4.15(<3.5)^{*}$ & $<3.0$ \\
\hline CAMP (mV) & $4.6,3.3,3.0^{* * *}$ & $6.1,4.4,4.2^{* * *}$ & $>6.0$ \\
\hline $\begin{array}{l}\text { Conduction velocity } \\
(\mathrm{m} / \mathrm{s})\end{array}$ & $31,22^{* *}$ & $39.7,44.4^{* * *}$ & $>50$ \\
\hline $\mathrm{F}$ wave latency (ms) & 67.37 & 43.2 & $<32$ \\
\hline \multicolumn{4}{|l|}{ Left Ulnar } \\
\hline Distal latency (ms) & & 3.75 & $<3.0$ \\
\hline CAMP $(\mathrm{mV})$ & & $5.8,3.7,3.4^{* * *}$ & $>6.0$ \\
\hline $\begin{array}{l}\text { Conduction velocity } \\
(\mathrm{m} / \mathrm{s})\end{array}$ & & $38.1,32.8^{* *}$ & $>50$ \\
\hline F wave latency (ms) & & $\mathrm{n} / \mathrm{a}$ & $<32$ \\
\hline \multicolumn{4}{|l|}{ Sensory } \\
\hline \multicolumn{4}{|l|}{ Sural } \\
\hline Distal latency (ms) & 4.9 & NR & $<4.3$ \\
\hline $\operatorname{SNAP}(\mu V)$ & 8.9 & NR & $>6$ \\
\hline $\begin{array}{l}\text { Conduction velocity } \\
(\mathrm{m} / \mathrm{s})\end{array}$ & 33 & NR & $>50$ \\
\hline \multicolumn{4}{|l|}{ Median } \\
\hline Distal latency (ms) & NR & NR & $<3.5$ \\
\hline $\operatorname{SNAP}(\mu V)$ & NR & NR & $>20$ \\
\hline $\begin{array}{l}\text { Conduction velocity } \\
(\mathrm{m} / \mathrm{s})\end{array}$ & NR & NR & $>50$ \\
\hline
\end{tabular}

Table 1: The nerve conduction study of case 1 and 2. CMAP: Compound Muscle Action Potential; m/s: Meter Per Second; $\mu$ V: Microvolt; ms: Milliseconds; mV: Millivolt; NL: Normal Limit; SNAP: Sensory Nerve Action Potential. *Study was done at an outside EMG lab. ${ }^{* *}$ Two numbers refer to conduction velocities recorded at routine stimulation segments. ***Three numbers refer to the amplitudes recorded from distally to proximally at common stimulation site. 


\section{Case 2}

A 65-year-old British white male patient developed dysphagia, slurred speech, ptosis, diplopia, and fatigable weakness 3 - 4 weeks after receiving influenza vaccine. MG was diagnosed based on positive AchR binding, modulating, and anti-striational antibodies, in addition to abnormal RNS study. Chest CT did not show thymic pathology. Bulbar symptoms improved significantly with IVIg, However, he kept getting worse with difficult walking, buckling knees, and foot drop a few months later. He was also diagnosed with CIDP based on the NCS (Table 1) and EMG. CSF analysis showed albuminocytologic dissociation (WBC $0 / \mathrm{cm}^{2}$ and protein $=161 \mathrm{mg} / \mathrm{dL}$ ). His limb weakness has been refractory to treatment, currently requiring 2 - $3 \mathrm{~g} / \mathrm{kg}$ IVIg every three weeks, mycophenolate $(1000 \mathrm{mg}$ twice a day), and prednisone $10 \mathrm{mg}$ daily. He recently got admitted to our service for plasmapheresis with a fair response

\section{Discussion}

Here we report two cases of concomitant CIDP and MG. While the combination of these two diseases was scarcely reported in both adults and children [3-7]. However, these earlier case reports did not discuss the response to treatment extensively. Moreover, all the previously reported cases were CIDP plus seropositive MG. To our knowledge, Case 1 is the first case report of CIDP coexisting with seronegative MG. In this case, MG was suspected clinically and tested positive on SFEMG. This emphasizes on the importance of considering the possibility of concomitant MG when a CIPD patient develops bulbar and/or ocular symptoms. While cranial and phrenic nerve involvement my occur in CIDP [8], the possibility of another autoimmune disease should always be entertained. Moreover, one should not rule out such possibility based only on negative AchR and MusK antibodies testing.

Our two cases initially presented differently. Case 1 presented with limb symptoms first and then developed bulbar and ocular symptoms six months later. Case 2 presented with bulbar symptoms and then developed the limb weakness a few months later. In both cases, the bulbar were more responsive to treatment than limb weakness.

\section{Conclusion}

The rare possibility of concomitant MG should still be entertained when CIDP patients develop bulbar or ocular manifesta- tions. To assess such possibility, SFEMG should be performed when routine serological testing for neuromuscular junction transmission disorders is negative. Although the main lines of treatment for MG and CIDP are similar, the response to treatment in our two cases was different with bulbar symptoms being more responsive to immunomodulatory treatment than limb symptoms.

\section{Acknowledgements}

Special thanks to the technicians and staff at the University of Alabama at Birmingham (UAB) Hospital Electrodiagnostic Laboratory and other health care providers at UAB who have professionally assisted our patients diagnostically and therapeutically.

\section{Conflict of Interest}

The authors report no conflict of interest.

\section{Bibliography}

1. Meriggioli MN and Sanders DB. "Autoimmune myasthenia gravis: emerging clinical and biological heterogeneity". The Lancet Neurology 8.5 (2009): 475-490.

2. Mathey EK., et al. "Chronic inflammatory demyelinating polyradiculoneuropathy: from pathology to phenotype". Journal of Neurology, Neurosurgery, and Psychiatry 86.9 (2015): 973985.

3. Kimura K., et al. "A case of myasthenia gravis in childhood associated with chronic inflammatory demyelinating polyradiculoneuropathy". Neuropediatrics 29.2 (1998): 108-112.

4. Tam DA and Chalmers A. "Chronic inflammatory demyelinating polyneuropathy and myasthenia gravis". Journal of Child Neurology 14.7 (1999): 478-479.

5. Mori M., et al. "Concomitant chronic inflammatory demyelinating polyneuropathy and myasthenia gravis following cytomegalovirus infection". Journal of the Neurological Sciences 240.1-2 (2006): 103-106.

6. Bolz S., et al. "CIDP, myasthenia gravis, and membranous glomerulonephritis - three autoimmune disorders in one patient: a case report". BMC Neurology 18.1 (2018): 113.

7. Quan W., et al. "Myasthenia gravis and chronic inflammatory demyelinating polyneuropathy in the same patient - a case report". International Journal of Neuroscience 128.6 (2018): 570-572. 
8. Nobile-Orazio E. "Chronic inflammatory demyelinating polyradiculoneuropathy and variants: where we are and where we should go". Journal of the Peripheral Nervous System 19.1 (2014): 2-13.

\section{Assets from publication with us}

- Prompt Acknowledgement after receiving the article

- Thorough Double blinded peer review

- Rapid Publication

- Issue of Publication Certificate

- High visibility of your Published work

Website: www.actascientific.com/

Submit Article: www.actascientific.com/submission.php

Email us: editor@actascientific.com

Contact us: +919182824667 